\title{
DEPTH OF FOCUS, EYE SIZE AND VISUAL ACUITY
}

\author{
Daniel G. Green, ${ }^{1}$ Maureen K. Powers ${ }^{2 *}$ and Martin S. Banks ${ }^{3}$ \\ 'Vision Research Laboratory. Neuroscience Building. University of Michigan. \\ Ann Arbor, MI 48109, U.S.A. \\ ${ }^{2}$ Department of Psychology. University of Washington. Seattle, WA 98195; \\ ${ }^{3}$ Department of Psychology. The University of Texas at Austin, \\ Austin, TX 78712, U.S.A.
}

(Received 19 December 1979)

\begin{abstract}
We develop formulas for calculating the approximate depth of focus of any eye. They show that the magnitude of depth of focus is inversely proportional to the size of the eye and to its visual acuity. One particular implication of these quantitative relations, which is supported by previous data from rats and human infants, is that small eyes with low acuity should have large depths of focus. We show that the observed relation between defocus and contrast sensitivity in rats in predicted by our formulas. We also analyze recent findings in human infants and show that they demonstrate a good correspondence between the improvement in accuracy of the accommodative response with age and the reduction in depth of focus (predicted from our formulas) as acuity and eye size increase over the same age range. Optical factors such as astigmatism, refractive error and chromatic and spherical aberration should have no effect on visual resolution unless they exceed the depth of focus of an eye. Thus, our arguments imply that these factors may be relatively unimportant in small eyes with low acuity.
\end{abstract}

\section{INTRODUCTION}

In a perfect optical system there is a single plane where the image of a given object is brought to optical focus. Moving the object toward or away from the system moves its focused image relative to that plane, hence blurring the image that falls on the plane. If an infinitely sensitive blur detector were placed in such a system's plane of optimal focus, then the distance through which an object could move before it appeared out of focus would be infinitely small; that is, the system's depth of focus would be infinitely small.

The eye is not a perfect optical system, however, and the retina is not infinitely sensitive to optical blur. The eye's depth of focus is therefore always finite. For example, in the adult human eye, Campbell (1957) found a depth of focus of about $\pm 0.43 \mathrm{D}$. That is, objects from $2.3 \mathrm{~m}$ in front of the eye to optical infinity would be in equivalent focus for an unaccommodated emmetropic adult human observer. Anything closer than that would be detectably blurred. Thus, acuity would be diminished if the eye did not accommodate in order to bring closer objects to focus.

Recent data, from our laboratories and elsewhere, suggest that other organisms may be less sensitive to optical blur than adult humans are. Consider the human infant, for example. Experiments from two different laboratories (Salapatek et al., 1976; Atkinson et al., 1977) measured the acuity of young infants (1-3 months of age) for gratings placed at widely varying

* Present address: Department of Psychology, Vanderbilt University, Nashville, TN 37420 , U.S.A. distances from the eyes. Neither group found any effect of target distance on acuity. These results imply that either (1) the infant accommodates accurately enough to bring the targets into focus or (2) the infant's depth of focus is greater than the adult's. The first of these seems less likely than the second, because the infant's ability to accommodate is limited: Banks (1980a) has recently confirmed the earlier finding (Haynes et al., 1965) that infants do not begin to accommodate accurately until 2-3 months of age.

Consider the rat as a second example. Powers and Green (1978) recorded the responses of ganglion cells to drifting gratings and placed spherical lenses of various powers in front of the eye. They found that the bursts of action potentials produced in response to the bars of the grating were essentially unaffected by lenses that were less than $\pm 14 \mathrm{D}$ in power. Because the rat is probably incapable of accommodating to such stimuli (Woolf, 1956), these results imply that. like the infant, the rat's depth of focus is greater than that of the adult human.

These observations about rat and infant vision led us to attempt a quantitative examination of the factors that contribute to depth of focus. In this paper we examine how optical factors interact with eye size and visual acuity in determining depth of focus.

We have two goals. The first is to provide simple formulas for calculating approximate depth of focus for any eve. We rely on paraxial ray optics and thin lens formulas to derive the relevant relations. Our second goal is to demonstrate that the combination of small size and low acuity produces large depth of focus. For this purpose we gather data from schematic eyes and studies of visual acuity in many organ- 
isms and apply our formulas to them. We also discuss data on accommodation in human infants that support the validity of our approach.

\section{GENERAL RELATIONS}

The precise depth of focus of any eye depends on many parameters, including axial length, pupil diameter, visual acuity, chromatic and spherical aberration and the type of stimulus being viewed. For the sake of simplicity, we will only consider the first three factors in this section. The others will be taken up in later sections.

Let us treat the eye as a single spherical refracting surface separating two media of refractive indices 1 and $n$ a common practice; see Davson, 1972). This reduced schematic eye is illustrated in Fig. 1. Point $a$ is the image of a distant point source (at optical infinity). It falls on the retina. Point $b$ is the image of a point source that has been brought closer to the eye so that its image is focused behind the retina. On the retina a blur circle of diameter $s$ is formed.

Now let the object $o$ approach the observer until an increase in blur is just detected. Let $s_{1}$ denote the diameter of the optical blur circle that is just detected as blurred by the neural detecting mechanism. We define the depth of focus of the eye, $\delta$ (Fig. 1) as the linear difference between the plane of best optical focus on the retina (where $s=0$ ) and the plane that produces the just-detectable blur circle on the retina (where $s=s_{1}$ ). To express this in diopters, we compute the change in power required to move the focal plane by an amount $\delta$. The quantitative relations are

$$
\begin{aligned}
\Delta D & = \pm(n / f-n /(f+\delta)) \\
& = \pm n \delta / f(f+\delta)
\end{aligned}
$$

- If $b$ fell in front of the retina instead of behind it, a blur circle would also be formed. For small changes it is safe to assume that the just-detectable amount of blur is the same whether the image falls in front or behind. For this reason, depth of focus is generally represented as $\pm D$.

$\uparrow$ We will deal with the problem directly in a later section, but for now let us assume that "acuity" and $s$ are related in a straightforward way. where $\Delta D$ is depth of focus in diopters, $n$ is the refractive index of the eye media, $f$ is the posterior focal length of the eye and $\delta$ is the distance from the retina to the optically focused image of the near object."

Examination of Fig. 1 shows that a simple relationship exists between the ratio of the diameter of the blur circle on the retina $(s)$ to the diameter of the pupil $(p)$ and the ratio of depth of focus to the sum of that $(\delta)$ distance and the focal length:

$$
s / p=\delta /(f+\delta)
$$

If we use the particular value of $s$ that represents the diameter of the just-detectable blur circle, then we can substitute equation (2) into the original equation for depth of focus to obtain the following:

$$
\Delta D=s_{1} n / p f
$$

where $s_{1}, p$ and $f$ are in units of distance. Equation (3) shows that depth of focus is inversely proportional to focal length and pupil size, and directly proportional to the diameter of the just-detectable blur circle on the retina.

To illustrate the use of equation (3) let us calculate the depth of focus of the adult human eye. We will assume that the adult eye is unable to distinguish between an optically focused point and a blur circle subtending an angle less than ' 1 ' of arc. This is probably an overestimate of the just-detectable blur circle, because foveal thresholds for spots less than about $3^{r}$ of arc depend only on light flux, independent of size; see Brindley, 1970 . If we use $f=22.9 \mathrm{~mm}$ and the conversion factor $5.0 \mu / \mathrm{min}$ (the appropriate figures for the reduced human eye; see Davson, 1972), equation (3) predicts a depth of focus of $\pm 0.10 \mathrm{D}$ for an observer with a $3 \mathrm{~mm}$ pupil. Several reports in the literature are consistent with this value (Hasegawa, 1952, cited by Oshima, 1958: $\pm 0.08 \mathrm{D}$; Ronchi and Fontana, 1975: $\pm 0.04 \mathrm{D}$; Charman and Whitefoot, 1977: $\pm 0.16 \mathrm{D}$ with $75 \%$ criterion). There are also papers that report higher values than we calculated (Campbell, 1957; $\pm 0.43 \mathrm{D}$; Green and Campbell, 1965: $\pm 0.25 \mathrm{D}$; Charman and Whitefoot, 1977: $\pm 0.47 \mathrm{D}$ with $98 \%$ criterion). We note, however, that

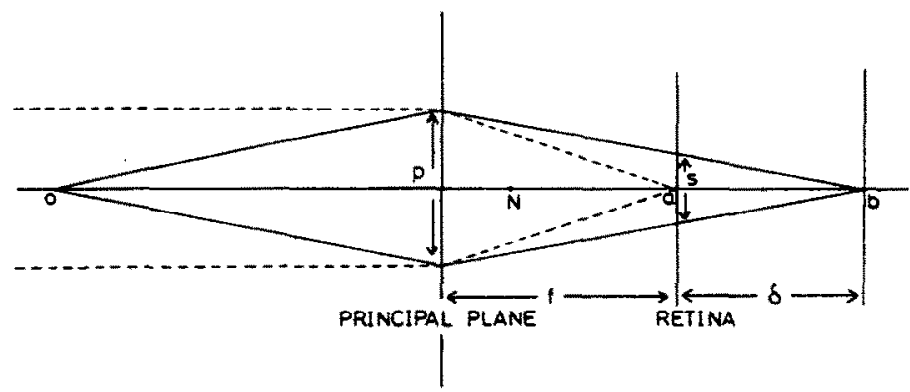

Fig. 1. Optical diagram. The eye is schematically represented by a single principal plane with focal length $(f)$. a circular aperture $(p)$ and nodal point $(N)$. Point $a$ is the image of a distant object and is assumed to fall on the retina. Point $b$ is the image of a near object $(0)$ and is focused a linear distance $\delta$ behind the retina, forming a blur circle of diameter $s$ upon the retina. 
the smallest and largest empirical values of adult human depth of focus differ by only about a factor of three. The discrepancies may be due to differences in stimuli used by the experimenters, or to differences in the criterion for blurriness used by the subjects (e.g. Charman and Whitefoot, 1977). We therefore conclude that by using equation (3) and assuming that the subtense of the minimally detectable blur circle is of the same order of magnitude as the minimally resolvable angle, one can obtain reasonable estimates of $\Delta D$ for the adult human eye.

\section{Depth of focus and eye size}

In order to illustrate how variations in the diameter of the eye might affect depth of focus, let us suppose for the moment that $s_{1}$, the diameter of the justdetectable blur circle on the retina, is constant across species. This is not true, of course, but it will allow us to consider the influence of optical factors independent of neural factors. Then let us make two assumptions in order to incorporate data from a number of species: (1) the posterior nodal distance (point $N$ to the retina in Fig. 1) is proportional to the axial length of the eye, and (2) the steady-state diameter of the pupil (under given retinal illumination) is also proportional to the axial length. Because light-gathering ability is determined by the numerical aperture (the ratio of pupil diameter to focal length), the second assumption is equivalent to assuming that this ability is similar across species. According to a recent review by Hughes (1977), both assumptions are reasonable (see his Figs $9 b$ and 10 ).

Formally, the first assumption can be written as

$$
f=k_{1} \cdot L
$$

where $f$ is focal length, $L$ is axial length and $k_{1}$ is the proportionality constant. Likewise, the second assumption can be written as

$$
p=k_{2} \cdot L
$$

where $p$ is pupil diameter and $k_{2}$ is another proportionality constant. Substituting equations (4) and (5) into equation (3) yields

$$
\Delta D=K s_{1} / L^{2}
$$

where $K=n / k_{1} \cdot k_{2}$. The interesting result is that with these two assumptions and a fixed just-detectable blur circle diameter, the depth of focus of an eye varies inversely as the square of the axial length of the eye. The consequences of this for a variety of animals are illustrated in Fig. 2a. The inverse square dependence is shown as a solid line of slope -2 in $\log -\log$ coordinates; the line has been placed so that it passes through the point: $\triangle D= \pm 0.1 \mathrm{D}, L=22.9 \mathrm{~mm}$. This is equivalent to selecting $K$ (in equation 6 ) so that the depth of focus of the adult human eye is $\pm 0.1 \mathrm{D}$, the value we calculated earlier. The solid line shows the depth of focus predicted by equation (6), assuming that $s$ and $K$ are equivalent to adult human foveal values, for a number of eyes whose axial lengths were obtained from the literature (Hughes, 1977; see caption for other references). This function illustrates that objects could approach small eyes quite closely without producing appreciable blurring of the retinal image.

Figure $2 \mathrm{a}$ demonstrates that if an eye's ability to detect blur were equivalent to that of the adult human fovea and its pupil diameter scaled directly with changes in size, then the eye's depth of focus would be inversely proportional to the square of its axial length. But acuity is considerably lower in many eyes than it is in the adult human fovea. Such eyes should be unable to detect the small changes in blur that the human eye can and, consequently, should have even larger depths of focus than Fig. 2 a predicts. The influence of acuity on depth of focus is examined in the next section.

\section{Depth of focus and visual acuity}

Because acuity is conventionally expressed in terms of resolvable angles, let us transform $s$ from linear dimensions on the retina into angular units. The angular subtense of a blur circle of diameter $s_{1}$ is

$$
\phi=\frac{180}{\pi} \cdot \frac{n s_{1}}{f}
$$

where $\phi$ is in degrees. Taking $n$ as $4 / 3$, solving equation (7) for $s_{1}$ and substituting into equation (3) yields

$$
\Delta D=17.45 \phi / p
$$

where $\phi$ is again in degrees, $p$ is pupil diameter in $\mathrm{mm}$, and 17.45 is a constant. Note that focal length is not involved in equation (8); this is because changes in focal length produce equivalent changes in both blur circle diameter and retinal image size.

Equation (8) shows that depth of focus depends only on the angular subtense of the just-detectable blur circle and on the diameter of the pupil. Pupil diameters are relatively easy to obtain, either from the literature or by direct measurement. But the subtense of the just-detectable blur circle has not been directly measured in any non-human eye, to our knowledge. A more readily available value is visual acuity, expressed in terms of the minimum resolvable visual angle. In order to relate acuity to the size of the justdetectable blur circle, we argue as follows. Imagine that the scene which is imaged on the retina is decomposed into a large number of weighted point sources. Now, consider the effect defocus has on the image of each point in the scene. As we have noted (Fig. 1), the blurred image of a point source is a circular disc of light. Consequently, blur circles from each point in the object will sum to produce the (blurred) image of the scene. Whether or not this degraded image appears to be blurred will depend on the structure of the scene (sharp edges and fine detail will suffer most from the effects of blur) and on the resolving capabilites of the detector (fine detail must first be resolved if one is to appreciate that it has lost clarity). We will 
deal with these complexities by assuming that detection of blur occurs when the size of the blur circle equals the size of the just-resolvable spatial detail in the scene. This assumption is certainly an oversimplification, but it is a conservative one: any errors that result from adopting it are likely to be in the direction of underestimating the depth of focus.

If $\phi$ is the angular subtense of the just-resolvable spatial target, we can rewrite equation (8) either in terms of minimal resolvable angle (in minutes of arc) or spatial frequency (in $\mathrm{c} / \mathrm{deg}$ ). We develop another approach in the appendix which allows us to relate acuity and depth of focus. The approach yields the relation:

$$
\Delta D=7.03 / p \cdot y
$$

where $v$ is visual acuity in c/deg. This equation is equivalent to equation (8) with the just-detectable blur diameter equal to $8 / 10$ of the stripe width of the cut-off spatial frequency.

Equation (8) can be used to estimate the depth of focus in any eye for which pupil diameter and visual acuity are known. It gives the same value for depth of focus of the adult human eye as we obtained from equation (3), if one assumes a high-frequency cutoff of $30 \mathrm{c} / \mathrm{deg}$ (Green and Campbell, 1965) and a $3 \mathrm{~mm}$ pupil. It also gives a reasonable estimate of the depth of focus of the rat's eye. According to Powers and Green (1978), the limit of resolution of rat ganglion cells is about $2.3^{\circ}$ of visual angle. For a pupil dia. of $3 \mathrm{~mm}$, equation (8) gives a depth of focus of $\pm 13.2 \mathrm{D}$,

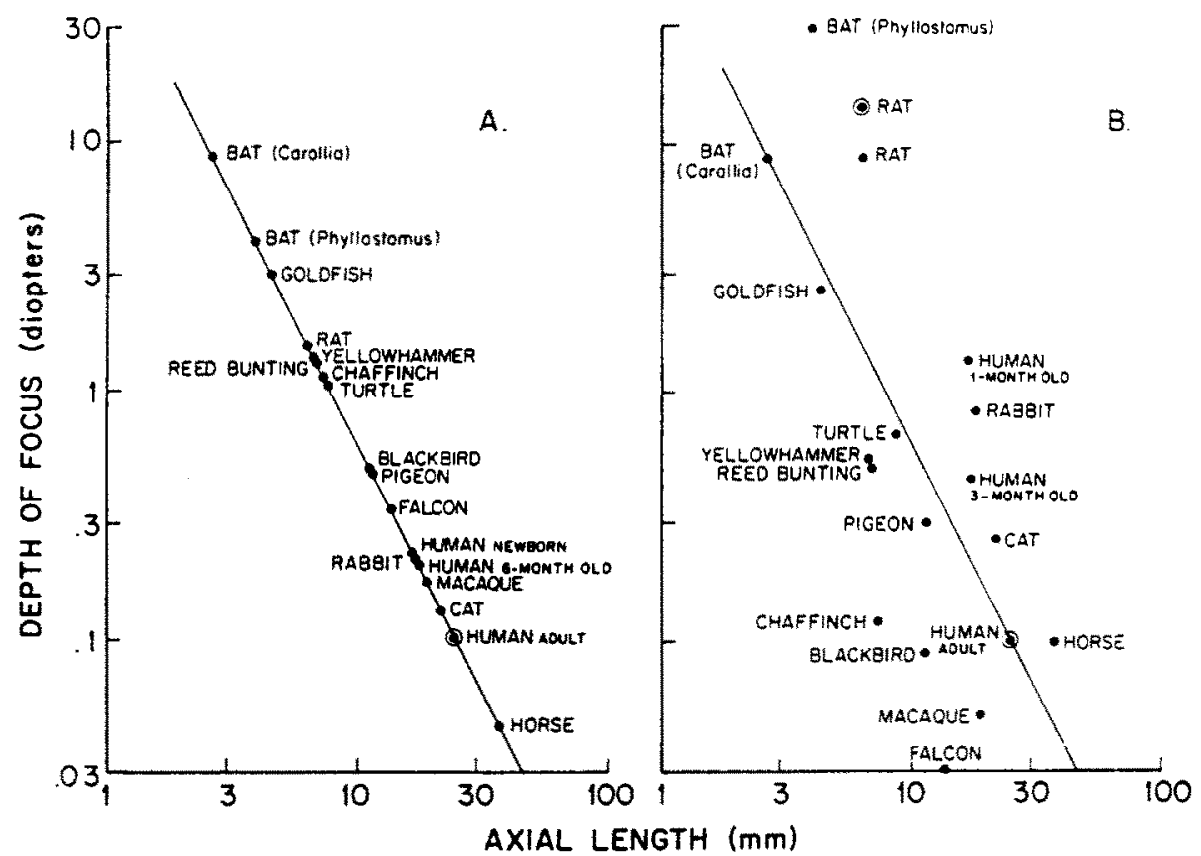

Fig. 2. Depth of focus predicted from eye size and visual acuity for a number of species. In (a) we have plotted the predictions from equation (6), assuming all organisms have visual resolving capacities equivalent to those of the adult human fovea. All fall on the solid line with slope -2 , because equation (6) shows that depth of focus is inversely related to the square of axial length. In (b) we have plotted the predictions for the same organisms, using equation (8) and behavioral measurements of acuity from the literature. (The circled point for the rat was calculated from the single ganglion cell data of Powers and Green (1978) for comparison.) We found axial length measurements in the following sources: bats: Suthers and Wallis (1970); goldfish: Charman and Tucker (1973); rat: Hughes (1979); yellowhammer, reed bunting, chaffinch, blackbird: (Donner, 1951); frog: DuPont and DeGroot (1976); turtle: Northmore, cited in Granda and Dvorak (1977); pigeon: Marshall et al. (1973); falcon: Lord (1956): human newborn and 6-month old: Larsen (1971); rabbit: Hughes (1972); macaque: Vakkur (1967), cited in Hughes (1977): cat: Vakkur and Bishop (1963), Vakkur et al. (1963) and Hughes (1976); human adult: Davson (1972); horse: Fig. $9 \mathrm{~b}$ in Hughes (1977).

Measurements of pupil diameter $\left(1 / 2\right.$ maximum or at $\left.10 \mathrm{~cd} / \mathrm{m}^{2}\right)$ were from the same sources, with the following exceptions : human infants: Banks (1980a); rabbit, macaque, horse: calculated from Fig. 10 in Hughes (1977); cat: Wilcox and Barlow (1975), value calculated from their measurements at $10 \mathrm{~cd} / \mathrm{m}^{2}$, assuming a round pupil; human adult: DeGroot and Gebhard (1952); falcon: R. Fox, personal communication. Behavioral measurements of visual acuity (minimum resolvable angle) were obtained from the following sources: bats: Suthers (1966); goldfish: Northmore and Dvorak (1979); rat: Wiesenfeld and Branchek (1976); yellowhammer, reed bunting. chaffinch, blackbird: Donner (1951); human infants: Atkinson et al. (1977), Banks and Salapatek (1978) and Allen (1979); pigeon: Nye (1968); macaque: Cowey and Ellis (1967) and DeValois et al. (1974); cat: Blake et al. (1974) and Bloom and Berkley (1977); human adult: Green and Campbell (1965); horse: Grzimek (1952); turtle: Dudziak (1955); falcon: Fox et al. (1976); rabbit: Van Hof (1967). 
compared to $\pm 14 \mathrm{D}$ that was actually required to reduce the response of ganglion cells by $20 \%$.

We have used equation (8) to calculate expected values of depth of focus in other organisms for which no verifying data exist, by using behavioral data on acuity and estimates of pupil diameter we found in the literature. Since the acuity and pupil diameter of a given eye vary with luminance, we chose values corresponding to a moderate photopic stimulus of $10 \mathrm{~cd} / \mathrm{m}^{2}$ whenever possible. These values are shown in Fig. $2 \mathrm{~b}$. The unverified values should be regarded as approximate because the data used to calculate them were often fragmentary. The solid line is the same as the one shown in Fig. 2a. Of the eyes represented in both figures, many show greater depth of focus when acuity, pupil diameter and eye size are considered (Fig. 2b) than when size alone is considered (Fig. 2a). The birds are notable exceptions, because of their high acuity and relatively large pupils.

\section{Depth of focus and accommodation in human infants}

One would expect that the magnitude and accuracy of accommodation needed to maintain optimal imagery in various eyes would depend on the magnitude of depth of focus in the unaccommodated eye. In this section, we present data from human infants that are consistent with this expectation. We draw together information about acuity, pupil diameter and the accommodative response to show that there is at least a correlation between the development of the ability to accommodate and the theoretical changes in depth of focus with age; namely, as depth of focus decreases, the accuracy of accommodation increases.

The accuracy of accommodation improves over the first months of life (Haynes et al, 1965; Banks, 1980a). In two experiments, Banks (1980a) measured infants' refractive state for targets presented at 25, 50 and $100 \mathrm{~cm}(+4,+2$ and $+1 \mathrm{D})$. If we plot his results as the change in refraction, called the "accommodative response" (in D), as a function of stimulus distance (also in D), we can characterize them by the slopes of their regression lines. Perfect accommodative response would yield a regression line with a slope of 1 . Slopes of the 1-month accommodation functions (regression lines) were 0.51 and 0.41 , whereas the average slopes of the 2-month functions were 0.75 and 0.79 , and of the 3 -month functions were 0.83 and 0.78 . The average adult slopes were 0.94 and 0.95 . Thus 1 -month olds do not accommodate very accurately, but by 2 to 3 months there is notable improvement.

We can use equation (9) to estimate depth of focus for the age groups and stimulus conditions used by Banks (1980a). His stimulus was a $30 \times 30$ random checkerboard with a relatively broad spatial frequency spectrum. Its space-average luminance was $8 \mathrm{~cd} / \mathrm{m}^{2}$. Pupil diameters were measured from infrared photographs of subjects viewing these stimuli. The average diameters were $4.2,4.6,4.6$ and $5.2 \mathrm{~mm}$ for 1 -, 2- and 3-month olds and adults, respectively. The highest spatial frequencies $1-, 2$ - and 3-month old

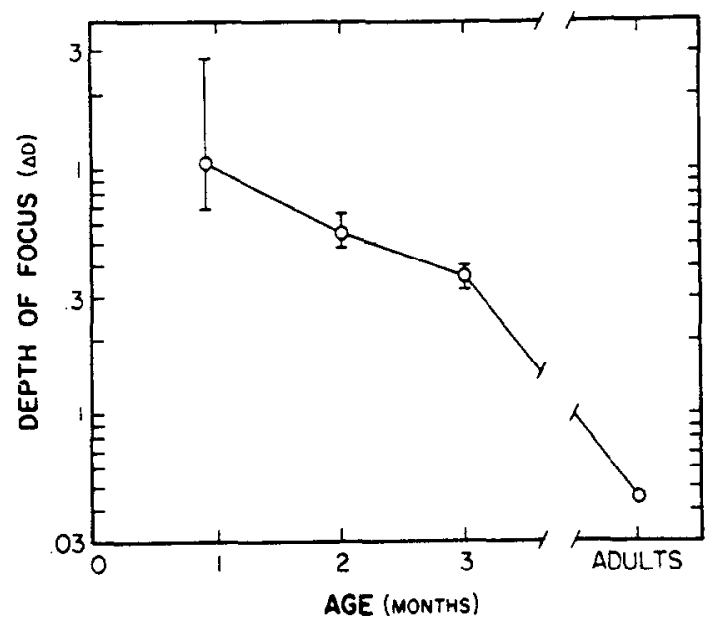

Fig. 3. Depth of focus values estimated from equation (9) for 1-, 2- and 3-month olds and adults. The points represent the average depths of focus calculated using acuity values from Allen (1979), Atkinson et al. (1977) and Banks and Salapatek (1978). The brackets represent the total range of depths of focus calculated using those acuity values.

infants can resolve range from 0.6 to $2.4 \mathrm{c} / \mathrm{deg}$ for 1 -month olds, 2.7 to $3.0 \mathrm{c} / \mathrm{deg}$ for 2 -month olds, and 4.0 and $4.5 \mathrm{c} / \mathrm{deg}$ for 3 -month olds. These acuity values were obtained in different laboratories, using stimuli of similar size and luminance (Atkinson et al., 1977; Banks and Salapatek, 1978; Allen, 1979). Substituting these pupil diameter and acuity values into equation (9) yields the values of depth of focus plotted in Fig. 3. We have drawn brackets at each age to represent the range of depths of focus predicted by the different acuity values. The solid lines connect the values obtained by using the average acuity values.

The next step is to relate our estimates of depth of focus to the measurements of accommodative response. Although it is not the only one we could have chosen, the approach we have selected is both simple and reasonable: we use depth of focus estimates of 1 -, 2- and 3-month olds and adults to predict the slopes of accommodation functions at those ages, and then we compare the predicted slopes with the slopes actually observed by Banks (1980a). We cannot make exact predictions of accommodative accuracy, however, because the resting point of accommodation is not yet known for infants. But it is possible to define a range of expected slopes in the following way. We first assume that the magnitude of accommodative error does not exceed the depth of focus. Then we calculate the slopes of the accommodation function for two cases: (1) where the resting point of accommodation is in the middle of the range of target distances used (i.e. between +1 and $+4 D$ ), and (2) where the resting point is at one or the other extreme of the range (i.e. at $+1 \mathrm{D}$ or at $+4 \mathrm{D}$ ). The dashed lines in Fig. 4 show the calculated values, which represent minimum (1) and maximum (2) estimates of slope. Figure 4 also shows the average empirical accommodative slopes for each group. Two points are plotted at each age to 


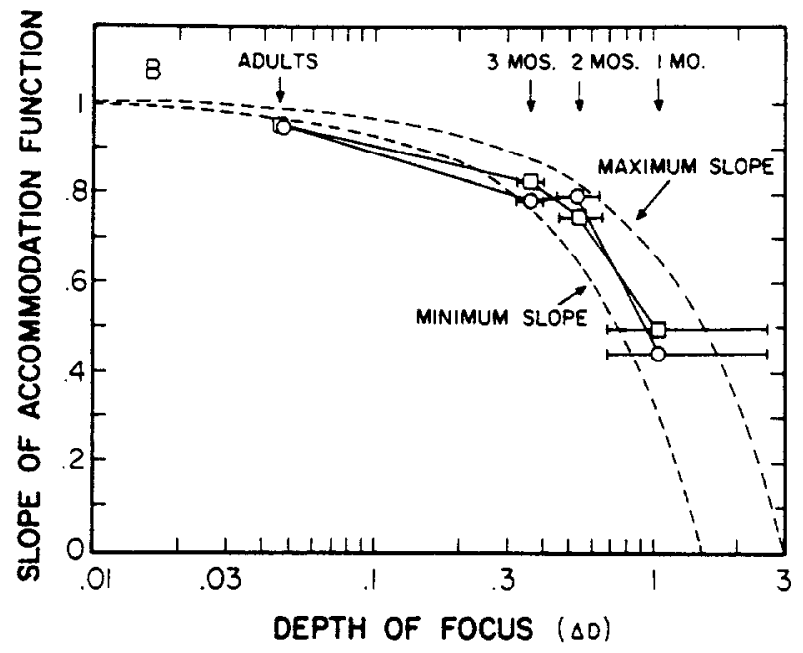

Fig. 4. Observed and predicted slopes of the accommodation functions for 1-, 2- and 3-month olds and adults. The broken lines represent the minimum and maximum predicted slopes. The data points represent the slopes obtained in Banks' (1980a) two experiments. Each point is positioned horizontally according to the depth of focus estimated for that age (see Fig. 3). The brackets represent the range of estimated depths of focus.

display the results of Banks' (1980a) two accommodation studies. The agreement between the observed and predicted slopes is good. Because the predicted slopes are entirely based on estimated depth of focus, this result implies that early infant accommodative development parallels age-related decreases in depth of focus.*

The above considerations clarify why acuity remains constant with viewing distance in young infants, even though they do not accommodate well: the depth of focus is sufficiently large that accommodative errors do not lead to a noticeable decline in visual acuity. Consider, for example, the findings of Salapatek et al. (1976): the acuity of 1-month olds was $1.5 \mathrm{c} / \mathrm{deg}$ (on the average) for target distances from 30 to $150 \mathrm{~cm}$, a range of $2.6 \mathrm{D}$. The depth of focus calculated from equation (9) for an acuity of $1.5 \mathrm{c} / \mathrm{deg}$ and a pupil diameter of $4.2 \mathrm{~mm}$ (Banks, $1980 \mathrm{a}$ ) is $\pm 1.4 \mathrm{D}$. Consequently, the minimal accommodation observed in 1 -month olds should be sufficient to keep visual acuity at its maximum for the range of distances tested.

\section{DISCUSSION}

We have examined the dependence of depth of focus on the size and visual acuity of eyes, and we have demonstrated that small eyes with low acuity are liable to have large depths of focus. The significance of this work lies in two domains. The first is practical. The formulas presented here allow any investigator to

* The observed value for adults is slightly outside the range of our predictions but the inclusion of chromatic and spherical aberration, diffraction due to the pupil, and other factors would inflate the predicted depth of focus and, consequently, the two points representing the adults would be shifted to the right. estimate the range of target distances over which stimuli should be in adequate focus for a variety of experimental subjects. The second is theoretical. Logic dictates that the magnitude of blur caused by various optical aberrations must exceed the depth of focus of an eye before the visual system can become aware that the image is blurred. Our work implies that small eyes with low visual acuity have large depths of focus, so they will be relatively unaffected by optical aberrations. For example, chromatic aberration is proportional to the focal power of the eye. But the depth of focus of the eye is proportional to the square of the power (equation 6), so for small eyes chromatic aberration is probably insignificant. To take a specific case: the rat's lens has about $3 \mathrm{D}$ of chromatic aberration between 487 and $617 \mathrm{~nm}$ (Millodot and Sivak, 1978); the inference that the depth of focus of the rat's eye is on the order of $\pm 10 \mathrm{D}$ (Fig $2 \mathrm{~b}$ ) means that the range of focal planes for different wavelengths is contained within the range where the rat should be unable to detect a difference in blurriness. This is not true of the human adult, where chromatic aberration between 487 and $617 \mathrm{~nm}$ $(0.75 \mathrm{D})$ is greater than depth of focus. Thus, for smaller eyes, chromatic aberration has a relatively smaller effect. Spherical aberration is smaller than chromatic aberration, at least in humans (Ivanoff, 1952), so its effect is also likely to be insignificant in eyes where depth of focus is large.

We have already seen that accommodative capacity increases as depth of focus decreases in growing human eyes. We speculate that eyes with large depth of focus may have poor accommodative capacity because with a large depth of focus, relatively large changes in an object's distance do not produce increases in perceived blur and, consequently, an accommodative change is not required. 
Refractive error also becomes significant only when it exceeds the depth of focus. The argument about whether or not retinoscopic measurements in small eyes yield artificially hypermetropic values (Glickstein and Millodot, 1970) may therefore be moot. For example, if the rat's eye has a depth of focus of $\pm 14 \mathrm{D}$ (Powers and Green, 1978), a refractive error of about the same size (Block, 1969; Glickstein and Millodot, 1970) still produces images that are in acceptable focus for the animal.

Finally, recent reports show that many young human infants have substantial astigmatic errors (Mohindra et al., 1978; Howland et al., 1978; reviewed by Banks, 1980b); Fulton et al., (1979), and large hyperopic errors (Cook and Glasscock, 1951; reviewed by Banks, 1980b). Our depth of focus calculations suggest that the perceptual consequences of such errors should be small; certainly less than they would be in adults. Indeed, behavioral measures of meridional variations in acuity among young infants with large astigmatisms reveal only relatively small deficits in the defocused meridia (Gwiazda et al., 1978; Howland et al., 1978; Held, 1979). Similarly, we would not expect the $+2 \mathrm{D}$ spherical error typical of newborn humans (see Banks, 1980b) to have substantial perceptual consequences.

Acknowledgements-The authors' research was supported by NIH grants EY00379 (D.G.G. and M.K.P.) and HD 12572 (M.S.B.). One of us (M.K.P.) also received financial support from NIH training grant EY07031 during preparation of the manuscript. We thank Walter Makous for comments on an earlier version.

\section{REFERENCES}

Allen J. (1979) Visual acuity development in human infants up to 6-months of age. Unpublished doctoral dissertation, University of Washington.

Atkinson J., Braddick O. and Moar K. (1977) Development of contrast sensitivity over the first 3 months of life in the human infant. Vision Res. 17, 1037-1044.

Banks M. S. (1980a) The development of visual accommodation during early infancy. Child Devel. in press.

Banks M. S. (1980b) Infant refraction and accommodation. In Electrophysiology and Psychophysics: Their Use In Ophehalmic Diagnosis. (Edited by Sokol S.). Little Brown, Boston.

Banks M. S. and Salapatek P. (1978) Acuity and contrast sensitivity in 1-,2- and 3-month-old human infants. Invest. Ophthal. 17, 361-365.

Blake R., Cool S. J. and Crawford M. L. (1974) Visual resolution in the cat. Vision Res. 14, 1211-1217.

Block M. T. (1969) A note on the refraction and image formation in the rat's eye. $V$ ision Res. 19, 705-711.

Bloom M. and Berkley M. A. (1977) Visual acuity and the near point of accommodation in cats. Vision Res. 17, 723-730.

Brindley G. S. (1970) Physiology of the Retina and Visual Pathway. Arnold, London.

Campbell F. W. (1957) The depth of field of the human eye. Optica Acta 4, 157-164.

Charman W. N. and Tucker J. (1973) The optical system of the goldfish eye. Vision Res. 13, 1-8.

Charman W. N. and Whitefoot H. (1977) Pupil diameter and the depth-of-focus of the human eye as measured by laser speckle. Optica Acta 24, 1211-1216.
Cook R. C. and Glasscock R. E. (1951) Refraction and ocular findings in the newborn. Am. J. Ophthal. 34. 1407-1419.

Cowey A. and Ellis C. M. (1967) Visual acuity of rhesus and squirrel monkeys. J. comp. Physiol. Psychol. 64, 80-84.

Davson H. (1972) The Physiology of the Eye. Academic Press, New York.

DeGroot S. G. and Gebhard J. W. (1952) Pupil size as determined by adapting luminances. J. opt. Soc. Am. 42 . 492-495.

DeValois R. L., Morgan H. and Snodderly D. M. (1974) Psychophysical studies of monkey vision-III. Spatial luminance contrast sensitivity tests of macaque and human observers. Vision Res. 14, 75-81.

Donner K. O. (1951) The visual acuity of some passerine birds. Acta Zool. Fenn. 66, 3-37.

DuPont J. and DeGroot P. J. (1976) A schematic dioptic apparatus for the frog's eye. Vision Res. 16, 803-810.

Dudziak J. (1955) Ostrosc widzenia u zolwia blotnego (Emys orbicularis L.) przy patrzenia w srodowisku powietrznym i wodnym. Folia Biol. 3, 205-228.

Fox R., Lehmkuhle S. and Westendorf D. H. (1976) Falcon visual acuity. Science 192, 263-265.

Fulton A. B., Dobson V. and Petersen R. A. (1979) Cycloplegic retractions in infants and young children. Invest. Ophthal. Suppl. p. 271.

Glickstein M. and Millodot M. (1970) Retinoscopy and eye size. Science 163, 605-606.

Granda A. M. and Dvorak C. A. (1977) Vision in turtles. In Handbook of Sensory Physiology. Vol. VII/5, Part A (Edited by Crescitelli F.), Springer, Berlin.

Green D. G. and Campbeil F. W. (1965) Effect of focus on the visual response to a sinusoidally modulated spatial stimulus. J. opt. Soc. Am. 55, 1154-1157.

Grzimek B. (1952) Versuche uber das Farbsehen von Pflanzenessern-I. Das farbige Sehen (und die Sehscharfe) von Pferden. Z. Tierpsychol. 9, 23-39.

Gwiazda J., Brill S., Mohindra I. and Held R. (1978) Infant visual acuity and its meridional variation. Vision Res. 18, 1557-1564.

Haynes H., White B. L. and Held R. (1965) Visual accommodation in human infants. Science 148, 528-530.

Held R. (1979) Development of acuity in normal and astigmatic infants. In Frontiers in Visual Science, (Edited by Cool S.) Springer, New York.

Hopkins H. H. (1955) The frequency response of a defocused optical system. Proc. R. Soc. A237, 91-103.

Howland H. C., Atkinson J., Braddick $O$. and French J. (1978) Infant astigmatism measured by photorefraction. Science 202, 331-333.

Hughes A. (1972) A schematic eye for the rabbit. Vision Res. 12, 123-138.

Hughes A. (1976) A supplement to the cat schematic eye. Vision Res. 16, 149-154.

Hughes A. (1977) The topography of vision in mammals of contrasting life style: Comparative optics and retinal organization. In Handbook of Sensory Physiology, Vol. VII/5, Part A. (Edited by Crescitelli F.). Springer, Berlin.

Hughes A. (1979) A schematic eye for the rat. Vision Res. $19,569-588$.

Ivanoff A. (1952) Les Aberrations de rOeil. Revue d'Optique. Paris.

Kulikowski J. J. (1976) Effective contrast constancy and linearity of contrast sensation. Vision Res. 16, 1419-1431.

Larsen J. (1971) The saggital growth of the eye-IV. Ultrasonic measurement of the axial length of the eye from birth to puberty. Acta Ophthal. 49, 873-886.

Levi L. (1974) Handbook of Tables of Functions for Applied Optics. CRC Press, Cleveland.

Lord R. D. Jr. (1956) A comparative study of the eyes of 
some falconiform and passeriform birds. Am. midl. Nat. $56,325-344$

Marshall J., Mellerio J. and Palmer D. A. (1973) A schematic eye for the pigeon. Vision Res. 13, 2449-2453.

Millodot M. and Sivak J. (1978) Hypermetropia of small animals and chromatic aberration. Vision Res. 18, 125-126.

Mohindra I., Held R., Gwiazda J. and Brill S. (1978) Astigmatism in infants. Science 202, 329-331.

Northmore D. P. M. and Dvorak C. A. (1979) Contrast sensitivity and acuity of the goldfish. Vision Res. 19. 255-261.

Nye P. W. (1968) The binocular acuity of the pigeon measured in terms of the modulation iransfer function. Vision Res. 8, 1041-1053.

Oshima S. (1958) Studies on the depth of focus of the eye. Jap. J. Ophthal. 2, 69-72.

Powers M. K. and Green D. G. (1978) Single retinal ganglion cell responses in the dark-reared rat: grating acuity. contrast sensitivity and defocusing. Vision Res. 18. 1533-1539.

Ronchi L. and Fontana A. (1975) Laser speckles and the depth of field of the human. Optica Acta 22, 243-246.

Salapatek P., Bochtold A. G. and Bushnell E. W. (1976) Infant visual acuity as a function of viewing distance. Child Devel. 47, 860-863.

Suthers R. A. (1966) Optomotor responses by echolocating bats. Science 152, 1102-1104.

Suthers R. A. and Wallis N. F. (1970) Optics of echolocating bats. Vision Res. 10, 1165-1173.

Vakkur G. J. and Bishop P. O. (1963) The schematic eye in the cat. Vision Res. 3, 357-381.

Vakkur G. J., Bishop P. O. and Kozak W. (1963) Visual optics in the cat, including posterior nodal distance and retinal landmarks. Vision Res. 3, 289-314.

Van Hof M. W. (1967) Visual acuity in the rabbit. Vision Res. 7. 749-751.

Wiesenfeld Z. and Branchek T. (1976) Refractive state and visual acuity in the hooded rat. Vision Res. 16, 823-827.

Wilcox J. G. and Barlow H. B. (1975) The size and shape of the pupil in lightly anaesthetized cats as a function of luminance. Vision Res. 15, 1363-1365.

Woolf D. (1956) A comparative cytological study of the ciliary muscle. Anat. Res. 124. 145-163.

\section{APPENDIX}

Depth of focus as estimated from modulation transfer functions

The performance of an ideal optical system limited only by diffraction can be specified by its modulation transfer function (MTF) (Levi, 1974). The MTF describes the loss of contrast sensitivity caused by an optical system for various spatial frequencies and aperture diameters. In this appendix we use theoretical MTF's to estimate depth of focus for various eyes. We show that using this more rigorous approach yields an equation for depth of focus (equation 9) that differs from the earlier, more intuitively derived equation (8), by less than 0.1 log unit in the constant term, and in no other way. Given the usual magnitude of experimental error in measuring visual acuity, this difference is insignificant, and the reader may use either equation (8) or equation (9) with equal confidence.

Green and Campbell (1965) demonstrated an inverse relationship between the spatial frequency of a grating and a

* It is now easy to relate equation (9) to equation $(8): s_{1}$. the just-detectable blur circle subtense, is equivalent to $8 / 10$ of the stripe width of $v$, the cut-off frequency. human observer's sensitivity to defocus. That is. a given amount of defocus reduced contrast sensitivity more for high spatial frequencies than for low frequencies. This relation would be predicted from the MTF of a defocused, diffraction-limited optical system (Hopkins, 1955: Levi, 1974). Hopkins' theoretical treatment of the diffractionlimited MTF included a complex equation which describes the contrast reduction caused by defocus at particular spatial frequencies. However, he found that one could ignore the influence of diffraction in many cases and thereby write a much simpler equation to describe the contrast reduction at various frequencies. Using our notation, his simpler equation states that:

$$
A_{d}(v) / A_{0}(v)=1-2 \pi^{2} \delta^{2} v^{2} i^{2}
$$

where $A_{0}(v)$ is the contrast at spatial frequency $v$ when no defocus is present, $A_{d}(v)$ is the contrast when a defocus error of magnitude $\Delta D$ is present, $i$ is wavelength, and $\delta$ is a linear measure of the defocus. Solving for $\Delta D$ and substituting for $\delta$ yields:

$$
\Delta D=15.71 \sqrt{1-M(v)} p \cdot y
$$

where $M(v)$ is equal to $A_{d}(v) / A_{0}(v)$. Here. as in equation (8), depth of focus is inversely proportional to spatial frequency.

Equation (11) yields an excellent approximation to the $\Delta D$ values yielded by Hopkins more rigorous, yet complicated formulation. To demonstrate this, we have calculated $\Delta D$ values using Levi's (1974) tables for the MTF of diffraction-limited lenses. We assumed. as others have (Hopkins, 1955: Charman and Whitefoot. 1977), that a 20\% reduction in contrast is just detectable. Figure 5 shows as solid lines the $\Delta D$ values estimated from the diffraction-limited MTF for $M(v)$ equal to 0.80 . (The values for one focal length, $20 \mathrm{~mm}$, are shown but, as suggested by earlier equations, equivalent values are obtained for other focal lengths.) The predictions of equation (11) are shown as dotted lines. The agreement is excellent when spatial frequency is low and when pupil diameter is large.

Equation (11) implies that depth of focus is only dependent on pupil diameter $(p)$, spatial frequency $(v)$, and the contrast reduction required to detect detocus $(1-. M(v))$, at least for low frequencies or large pupils. But most visual targets have a broad spectrum of spatial frequencies. so we must consider the frequency band upon which blur detection is most dependent. Assuming that the criterion conirast reduction $(1-M(v))$ is at least approximately constant for various spatial frequencies, depth of focus in a diffraction-limited system is smallest for the highest detectable frequency (this is certainly valid for eyes with relatively low visual acuity). Thus, we will make the simplifying yet reasonable assumption that blur detection is dependent on noting contrast reductions in the region near the highfrequency cutoff or resolution limit We implement this assumption by stating that $v$ in equation (11) represents the cutoff spatial frequency in c/deg even though a frequency band slightly below the cutoff value would probably be more reasonable. With this assumption. equation (11) can be generalized to broad spectrum stimuli.

The contrast reduction $(1-M(v))$ required for detection of defocus is not known, but if we accept a value of 0.2 , as we did before (and 0.2 is apparently reasonable (Kulikowski, 1976)), equation (11) becomes equation (9) which was given but not derived on p. 830 .

$$
\Delta D=7.03 / p \cdot v
$$

where $v$ now represents the cutoff spatial frequency in $\mathrm{c} / \mathrm{deg}$. Doubling the criterion for detecting contrast reduction to 0.4 would change the numerator of equation (9) to $7.03 \cdot \sqrt{2}$ or 9.94 . This illustrates that the depth of focus values vary with the square root of criterion and are therefore not as affected by changes in criterion as by changes in pupil diameter and visual acuity." 


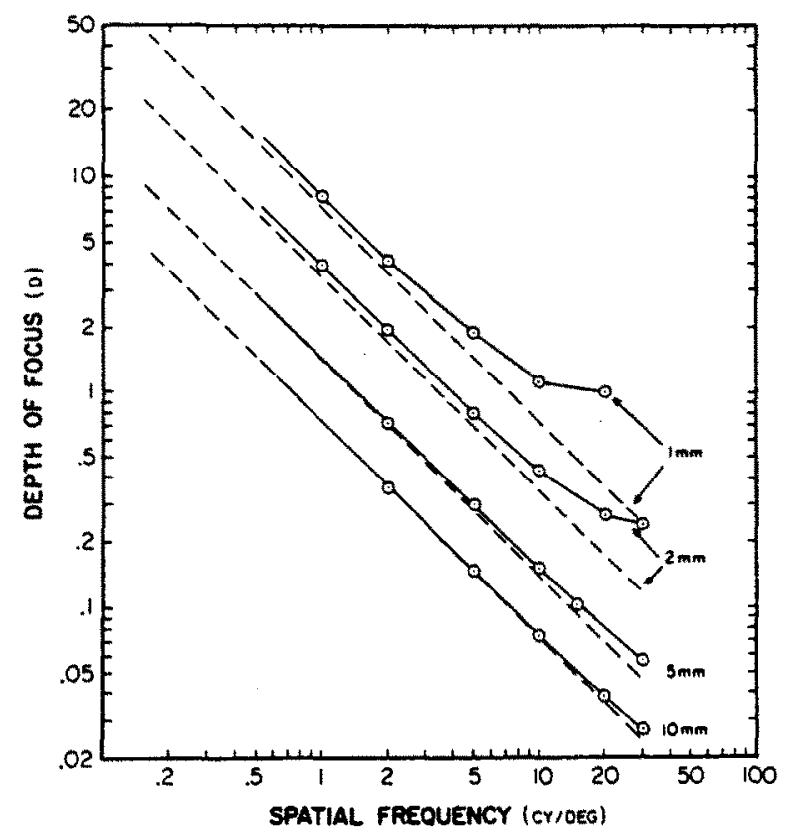

Fig. 5. Depth of focus as a function of spatial frequency and pupil diameter. The solid lines show depth of focus values calculated from the diffraction-limited MTF (Levi, 1974) for M(v) equal to 0.80. These values are plotted against spatial frequency in c/deg. Each line shows the values for the pupil diameter stated on the right. The broken lines show the values obtained using equation (11), a simpler formulation which does not incorporate the effects of diffraction.

This formulation once again suggests that depth of focus is not directly dependent on focal length. One should keep in mind, however, that this does not imply that eye size is unimportant given the correlation between pupil size and axial length (Hughes, 1977). In fact, equation (9) could be written as

$$
\Delta D=7.03 /\left(k_{2} L\right) v
$$

to emphasize the inverse relation between eye size and depth of focus.

The derivation of equation (9) was simplified by con- sidering an ideal optical system free from diffraction and such defects as chromatic and spherical aberration. The curves of Fig. 5 demonstrate that diffraction can signif. cantly enhance depth of focus at high spatial frequencies when the pupillary aperture is small. Chromatic and spherical aberration also increase depth of focus in the adult human eye, so equation (9) (and the curves of Fig. 5) should underestimate the adult human's depth of focus. These aberrations, however, do not significantly affect depth of locus at low spatial frequencies. Consequently. their contribution can be ignored for many organisms. 